\title{
Grey Correlation Analysis between Urbanization Rate and Household Consumption Structure within Liaoning Province
}

\author{
Xiao-Tong LIANG ${ }^{1, a}$, Wei-Hua YANG ${ }^{2, b}$ \\ ${ }^{1}$ China, Liaoning Province, Dalian Polytechnic University \\ ${ }^{2}$ China, Liaoning Province, Dalian Polytechnic University \\ aliangxiaotong919@sina.com, bjasonyangwh@sina.com
}

Keywords: Urbanization rate, Household Consumption, Grey Correlation Analysis.

\begin{abstract}
The paper analyzed the level of urbanization and the trends of household consumption within Liaoning Province. Three sets of corresponding data, included the urbanization rate, rural residents' average propensity to consume and urban residents' average propensity to consume, were applied to the econometric analysis. Then, the paper made an conclusion that the household consumption has an positive promotion to the development of urbanization. Further more, Grey Correlation Analysis was applied to analyze the correlation of urban and rural household consumption between eight categories of consumption, found that five of the eight categories of consumption were closely related to rural household consumption. This showed that rural household consumption had made a great contribution to improve the level of urbanization
\end{abstract}

\section{Introduction}

There are many researches about the relationship between urbanization and other economics indicators, such as carbon dioxide (CO2) emissions, energy consumption, real output (GDP), the square of real output (GDP2), trade openness, urbanization, and financial development.[1] some researches examined the impact of natural gas consumption, output, and urbanization on $\mathrm{CO} 2$ emission in China.[2] Unlike previous studies, some paper empirically investigates the impact of urbanization on energy consumption and $\mathrm{CO} 2$ emissions with consideration of provincial differences. [3]We would find that almost all the researches were concentrate on the relationship between urbanization and carbon emission, they talked about environmental issues. But from the household consumption and urbanization point of view, they were almost did not exist.

According to thee Liaoning Provincial Statistical Yearbook data ${ }^{[4]}$, compared to the early stages of Reform and Opening, the urbanization rate of Liaoning Province has been reached up to 67.5\%. This shows that Liaoning Province has been basically realized urbanization.

Although China has a fast growth in the area of GDP in recent years, the consumption rate has a downward sloping trend. This implied that problems in the field of consumption should be concerned more. From year 2010, several scholars focused on the Empirical Study of the relationship between urbanization and household consumption. All of them were identical, this paper made a distinction, concentrating on the different impact of rural and urban household consumption on urbanization respectively, measuring the degree of the impact by using the method of Grey Correlation Analysis.

\section{Granger Causality Test of Residents’ APC the Urbanization Rate}

\section{Data Sources}

Three main indicators including the urbanization rate $(X)$, rural residents' average propensity to consume(Y) and urban residents' average propensity to consume(Z) were selected from Liaoning Provincial Statistical Yearbook from 1980 to 2014. Then, the paper found the logarithmic form of the 3 variables in order to avoid the effect of heteroscedasticity. 


\section{Stability Test}

The sequence of lnx, lny and lnz were greater than 1\%-level ADF value, so it came to concluded that the sequence of lnx, lny and lnz has unit root. Further, for the first difference unit root test, the first difference of the series is stationary, so that the three sequences are integrated for first difference.

Table 1 Stationary test of original sequence and first difference test

\begin{tabular}{|c|c|c|c|}
\hline sequnece & t-statistics & 1\%-level ADF value & results \\
\hline $\ln x$ & 0.066 & -3.56 & unstable \\
\hline $\ln y$ & -2.58 & -3.56 & unstable \\
\hline $\ln z$ & -2.47 & -3.56 & unstable \\
\hline Dlnx & -5.68 & -3.56 & stable \\
\hline Dlny & -6.47 & -3.56 & stable \\
\hline Dlnz & -6.24 & -3.56 & stable \\
\hline
\end{tabular}

\section{Cointegration Test}

The paper made cointegration test based on the residual coefficients, found that the residuals of the three variables were stationary series, which ensured that the empirical analysis was effective.

\section{Granger Causality Test}

The Granger causality test shows that $\ln Y$ is the Granger cause of $\ln X$ at $10 \%$ significance level, $\ln \mathrm{Z}$ is the Granger cause of $\ln \mathrm{X}$ at $10 \%$ significance level. Therefore, the paper would make a more detailed discussion about if the rural consumer or the urban consumer had a greater contribution on the development of urbanization.

Table 2 Granger causality test of sequence $\ln \mathrm{X}, \ln \mathrm{Y}$ and $\ln \mathrm{Z}$

\begin{tabular}{lccc}
\hline Pairwise Granger Causality Tests & & & \\
Sample: 19802013 & & & \\
Lags: 2 & Obs & F-Statistic & Probability \\
Null Hypothesis: & 33 & 18.7570 & $7.8 \mathrm{E}-06$ \\
\hline LNZ does not Granger Cause LNX & & 0.80896 & 0.45583 \\
LNX does not Granger Cause LNZ & & 2.86555 & 0.07438 \\
LNY does not Granger Cause LNX & & 1.18790 & 0.32030 \\
\hline
\end{tabular}

\section{Analysis of the Impact of Urbanization on Consumption Structure}

\section{Data Selection and Classification of Consumer Goods and the Residents}

According to the principle of China Statistical Yearbook in the Urban Households Base, there're eight categories in consumer goods, including food, clothing, housing, household equipment goods and services, health care, transportation and communications, education, culture and entertainment services and miscellaneous goods and services. Using the data from 2005 to 2013, the paper calculated the proportion of the eight kinds of consumer goods in the total household consumption respectively, then the consumer goods per capita spending forms. The paper divided the residents into urban and rural population, then calculate the proportion of the rural and urban population in the whole population respectively. 


\section{The Process of Grey Correlation Matrix’s Calculation}

The proportion of rural population was designated as Y1, Y2 and regarded as the parent sequence of the matrix. The proportion of food, clothing, household equipment goods and services, health care, transportation and communications, education, culture and entertainment services and miscellaneous goods and services in consumer goods in the total consumption expenditure were designated as $\mathrm{X} 1, \mathrm{X} 2, \mathrm{X} 3, \mathrm{X} 4, \mathrm{X} 5, \mathrm{X} 6, \mathrm{X} 7, \mathrm{X} 8$ and regarded as the promoter sequence of the matrix.

Firstly, making a initial treatment to the original value; secondly , calculating the differencing sequence, that is $\Delta \mathrm{YnXk}=\mid \mathrm{Yn}$-Xk $\mid$; thirdly, finding the biggest difference between the two determined minimum difference for each time series the maximum value of max series had minimum value min papyemXlkad speaificatioxlthis 0.458 ,

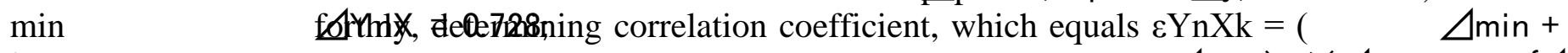
$\xi$ correlation coefficient of this paper was as follows:

$$
\begin{aligned}
& \varepsilon \mathrm{Y}_{1} \mathrm{X}_{\mathrm{k}}=(0+0.5 * 0.458) /\left(\Delta \mathrm{Y}_{1} \mathrm{X}_{\mathrm{k}}+0.5 * 0.458\right)=0.229 /\left(\Delta \mathrm{Y}_{1} \mathrm{X}_{\mathrm{k}}+0.229\right) \\
& \varepsilon \mathrm{Y}_{2} \mathrm{X}_{\mathrm{k}}=(0+0.5 * 0.728) /\left(\Delta \mathrm{Y}_{1} \mathrm{X}_{\mathrm{k}}+0.5 * 0.728\right)=0.364 /\left(\Delta \mathrm{Y}_{1} \mathrm{X}_{\mathrm{k}}+0.364\right)
\end{aligned}
$$

Finally, find the correlation, correlation is equal to $1 / \mathrm{N} * \sum \varepsilon \mathrm{Y}_{\mathrm{n}} \mathrm{X}_{\mathrm{k}}$. The correlation coefficient of parent sequence and the promoter sequence would be produced in the final results. The correlation coefficient is closer to 1, indicating that the parent sequence and the promoter sequence has strong correlation. The correlation coefficient is closer to 0 , indicating that the parent sequence and the promoter sequence has weak correlation. The corresponding results of this paper are as follows:

$$
\mathrm{R}=\begin{array}{lllllllllll}
r Y_{1} X_{1} & r Y_{1} X_{2} \ldots \ldots & r Y_{1} X_{8} & 0.743 & 0.818 & 0.757 & 0.566 & 0.550 & 0.676 & 0.771 & 0.830 \\
r Y_{2} X_{1} & r Y_{2} X_{2} \ldots . . & r Y_{2} X_{8} & 0.934 & 0.710 & 0.874 & 0.584 & 0,784 & 0.649 & 0.833 & 0.784
\end{array}
$$

\section{The Results of Grey Correlation Matrix}

The result of grey correlation matrix shows that urban households has larger proportion than rural households in clothing consumption. Because, along with the accelerated pace of urbanization, a large number of young workers flooded into the city, they are unconventional and self-reliant, with a greater consumption ability to become the main force of clothing consumption. In addition, most elderly and children were in rural areas, the elder are thrift and children were affected by the income limit. For food, housing, medical care, household equipment goods and services, education, culture and entertainment services, urban households have larger proportion than rural households. In recent years, rural residents had raised their wage levels and rural residents for food consumption had optimistic expectations, therefore, increasing the current consumption for food. Owing to the coverage and popularization of rural health insurance is not wide enough for medical care in rural areas. In recent years, health care cost continued to rise. Physiological factors of the elder and children in rural areas decided that rural residents had a large health care demand. In the area of educational and cultural entertainment services, attributing to the development of television, Internet and other advanced technology, which reversing rural residents' inherent ideology. Rural residents were beginning to realize the vital importance of education and paying more attention to spiritual enjoyment. Thus, the demand for educational cultural and recreational services had increased.

\section{Summary}

The average consumption level of urban and rural residents and the urbanization rate had a direct relationship of cointegration. Granger causality test showed that the average consumption of urban and rural residents had in some way improved the urbanization rate. 
Compared to urban residents, the rural population proportion and the food, housing, medical care, household equipment goods and services, cultural and recreational services had closely associated, indicating that the rate of urbanization and rural consumption are more relevant, rural households had greater influence on urbanization.

\section{Acknowledgement}

Major science and technology platform project of Liaoning Province universities, and open project of National Marine Food Engineering Research Center provided funding for this research. The project number is 2012FU125X03.

\section{References}

[1]Eyup Dogan, Berna Turkkul, CO2 emissions, real output, energy consumption, trade, urbanization and financial development: testing the EKC hypothesis for the USA, J. Environmental Science and Pollution Research. 23 (2016) 1203-1213.

[2] Sakiru Adebola Solarin, Hooi Hooi Lean, Natural gas consumption, income, urbanization, and CO2 emissions in China and India, J. Environmental Science and Pollution Research. 17 (2016) $1-13$.

[3] Qiang Wang, Shi-dai Wu, Exploring the relationship between urbanization, energy consumption, and CO2 emissions in different provinces of China, Renewable and Sustainable Energy Reviews, Fuzhou, 2016, pp. 1563-1579.

[4] Information on http:// www.ln.stats.gov.cn/ 